\title{
A Review on Facial Emotion Recognition and Classification Analysis with Deep Learning
}

\author{
Asha Jaison ${ }^{1}$ and C Deepa ${ }^{2}$ \\ ${ }^{1} M$. Phil Research Scholar, Department of Information Technology, Sri Ramakrishna \\ College of Arts and Science, Coimbatore, Tamilnadu, India \\ ${ }^{2}$ Associate Professor Department of Information Technology, Sri Ramakrishna College of Arts \\ and Science, Coimbatore, Tamilnadu, India \\ Corresponding author email: ashajaison@gmail.com,deepapkd@gmail.com
}

\begin{abstract}
Automatic face expression recognition is an exigent research subject and a challenge in computer vision. It is an interdisciplinary domain standing at the crossing of behavioural science, psychology, neurology, and artificial intelligence. Human-robot interaction is getting more significant with the automation of every field, like treating autistic patients, child therapy, babysitting, etc. In all the cases robots need to understand the present state of mind for better decision making. It is difficult for machine learning techniques to recognize the expressions of people since there will be significant changes in the way of their expressions. The emotions expressed through the human face have its importance in making arguments and decisions on different subjects. Machine Learning with Computer Vision and Deep Learning can be used to recognize facial expressions from the preloaded or real time images with human faces. DNN (Deep Neural Networking) is one among the hottest areas of research and is found to be very effective in classification of images with a high degree of accuracy. In the proposed work, the popular dataset CK+ is analysed for comparison. The dataset FER 2013 and home-brewed data sets are used in the work for calculating the accuracy of the model created. The results are obtained in such a way that DCNN approach is very efficient in facial emotion recognition. Experiments and study show that the dataset, FER 2013 is a high-quality dataset with equal efficiency as the other two popular datasets. This paper aims to ameliorate the accuracy of classification of facial emotion.
\end{abstract}

KEY WORDS: FACIAL EMOTION RECOGNITION (FER), DEEP CONVOLUTIONAL NEURAL NETWORK (DCNN), OPENCV (OPEN-SOURCE COMPUTER VISION LIBRARY), VGG 16.

\section{INTRODUCTION}

Humans use a facial expression for non-verbal communication to show their emotional states. By observing someone's face, we can assume their emotion. However, FER (Fasel \& Luettin, 2003)is still a challenging research area in various applications related to computer vision. But undoubtedly the application includes FER

Biosc Biotech Res Comm P-ISSN: 0974-6455 E-ISSN: 2321-4007

\section{crossef}

Identifiers and Pagination

Year: 2021 Vol: 14 No (5) Special Issue

Pages: 154-161

This is an open access article under Creative

Commons License Attribn 4.0 Intl (CC-BY). DOI: http://dx.doi.org/10.21786/bbrc/14.5/29 for security (Butalia, et al., 2012), FER for mindset identification (Mandal, et al., 1998), FER for psychology aid, crime detection (Polikovsky, et al., 2009), Intelligent Tutoring System (Kumari, et al., 2015), Driver Fatigue Detection (Zhang \& Zhang, 2006), Music based on Mood analysis (Dureha, 2014). Emotion refers to the internal feelings of human, through which they communicate their emotional states and intentions. The goal of this work was to classify the human emotions. The Conventional feature extraction and classification has complex computations for achieving high recognition rate. The proposed method used deep learning model to classify facial emotions from the dataset or real time image. The deep learning technique outperforms other conventional methods by its capability of handling large dataset and capability of faster computation. Deep learning consists of neural
Article Information

Received: $24^{\text {th }}$ Jan 2021 Accepted after revision: $26^{\mathrm{h}}$ Mar 2021 
network model, in which the connected neurons will act as inputs and outputs. This model is aimed to test and train the proposed system.

The paper is organized as follows.

Section I describes the introduction part of Facial Expression Recognition, Section II describes the Classes of Emotion and its description, Section III will help us to learn about Conventional approaches to FER, Section $\mathrm{V}$ deals with the study of FER using Deep Learning approach, Section VI deals with Results and Conclusion and finally, references are included.

II. Major Classes of Emotion: Emotion is the primary motivation for every human being. Each emotion has its specific distinctive signals in brain. The changes in neural stimulation results in activation of emotions. Emotions are dependent on the situation and present condition of a human. Emotions get normally reflected on human face in the form of various expressions. The basic expressions on the face are happy, anger, fear, sad, surprise and disgust (Darwin, 1872) (Ekman, et al., 1971) (Albert, 2008). Each emotion has got its characteristics (Shoayuan, et al., 2019) (Lucey, et al., 2010). The basic expressions that are mentioned shall be depicted as shown in Figure:1.

Figure 1: Universally accepted seven human emotions: Angry, Disgust, Fear, Happy, Neutral, Sad, Surprise

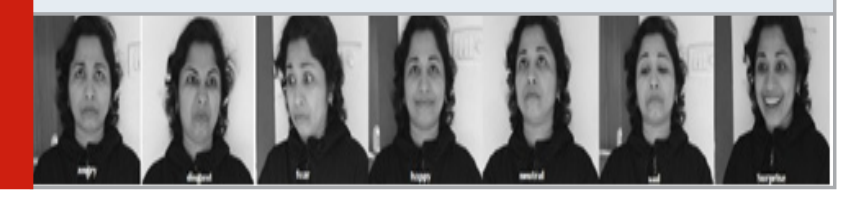

Figure 2: Flow diagram of Facial Emotion Recognition

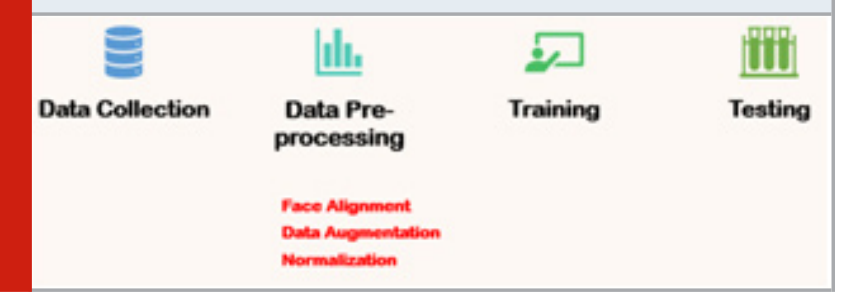

III. Steps of Fer in Conventional and Deep Learning Approach: The first step in FER is image pre-processing, one can follow any of the processing techniques like Wavelet Transform (Haiyang, 2011), Discrete Cosine Transform, Colour Normalization Methods, Binarization (Anitha \&t Radha, 2010), Histogram Equalization (Anila \& Devarajan , 2012) etc. The second step is Detecting face, that is to locate one or more faces in the given image and the identified face is bounded within a square box. In face component detection step, we detect the ROI for eyes, eyebrow, nose, cheeks, mouth forehead, ears etc. Various feature extraction techniques can be used for extracting features from ROIs.

The techniques include Local Binary Pattern [LBP] (Ojala, et al., 1996), Principal Component Analysis [PCA] (Turk \&t Pentland, 1991), Linear Discriminant Analysis
[LDA] (Belhumeur, et al., 1997), Local Gradient Code [LGC] (Tong, et al., 2014), Independent Component Analysis [ICA] (Bartlett, et al., 2002), Local Directional Pattern [LDP] (Jabid, et al., 2010) etc. After feature extraction classification can be done. The classifiers classify the features based on the classification methods used. Traditional Classifiers like Euclidean Distance, N Neighbourhood etc can also be used for classification. The most popular methods used are Hidden Markov Model (HMM) (Kumar \& Gupta, 2015), Neural Network (NN), Support Vector Machine (SVM) (Eschbach, et al., 2014), AdaBoost etc.

Figure 2 shows the flow of Facial Emotion Recognition. The data is collected from the publicly available datasets like $\mathrm{CK}+$, JAFFE etc or sequence of images can be given as input. The pre-processing step includes removal of noise from the image, Face Alignment, Data Augmentation, Normalization etc is done. Later it is trained and tested.

Deep Learning Based Facial Emotion Recognition Approach: From this section, different approaches towards FER based on Deep Learning can be learned. (Chen, et al., 2017) (Kumar, et al., 2019) proposed a new CNN structure for FER, and they used CK+ and JAFFE databases for experiments. The detected faces are cropped using OpenCV library and the facial features are extracted with CNN using deep learning. LENet, the CNN network structure for FER is introduced with two pooling layers, four convolution layers and 2 fully connections layers (Shamsolmoali, et al., 2018). The model is trained and tested over CK+ Database and for validation JAFFE database is used. The feature is extracted using Deep Neural Network and used SoftMax for classification.

(Sun, et al., 2018) introduced a method for Facial Emotion Recognition, they divided face into three active regions, and these active regions are optimized. CNN is trained for all the three active regions and is trained to extract the facial features and classify the expressions. The experiments were done over 3 databases CK+ (University, 2021), JAFFE (Openaire, 1998)and NVIE (Wang, et al., 2010). The Speed of recognition is done in 0.01 Seconds. (Kumbhar, et al., 2017) proposed a methodology for tracking and detecting faces from a real-time HD Video. The Open libraries that are provided by Open CV (OpenSource Computer Vision Library) are used for tracking and detecting faces. The feature is selected with the help of HAAR classifier and trained. They implemented this on Raspberry -Pi.

The proposed work mainly makes a study and an analysis on the accuracy rate obtained towards the different data sets trained and tested. The commonly used Viola - Jones Algorithm (Wang, 2014) is used for face detection in the experiment. The major step includes i) Image Pre-Processing ii) Feature extraction from the Grayscale facial Images iii) Feature extraction from LBP facial images iv) Weighted fusion of different outputs. In the Pre-processing stage, face is detected from the benchmarked facial emotion dataset or from a live 
capture. The next stage is rotation correction, this issue can be resolved by applying the rotation transformation matrix as follows:

$\left(R x^{\prime}, R y^{\prime}, 1\right)=\left[\begin{array}{lll}R x & R y & 1\end{array}\right]\left[\begin{array}{ccc}\operatorname{Cos} \theta & \operatorname{Sin} \theta & 0 \\ -\operatorname{Sin} \theta & \operatorname{Cos} \theta & 0 \\ 0 & 0 & 1\end{array}\right]$

where $(\mathrm{Rx}, \mathrm{Ry})$ represents the original coordinate in face input image and $\left(\mathrm{Rx}^{\wedge}, \mathrm{Ry} \mathrm{y}^{\prime}\right)$ is the $(\mathrm{x}, \mathrm{y})$ coordinates after Rotation Transformation, $\theta$ represents the rotation angle. Rotation angle formed by making the line segment between the centre of eyes to the horizontal axis. LBP (Chan, et al., 2007) (Turabzadeh, et al., 2017) (Shan, et al., 2009) helps to identify the properties of individual parts of the input image. (Ojala, et al., 2002) introduced LBP algorithm as a combination of structural and statistical implementation on texture images with monotonic grayscale. Figure 3 illustrates the basic functioning of LBP.

LBP descriptor is used for capturing texture information of the given image and is calculated by comparing its value with adjacent pixels. The left part of the figure illustrates the actual pixel values and right part indicates the corresponding LBP coding, and the central pixel is calculated with binary coding.

After the encoding of pixels are completed, LBP can be calculated with the equation.

$$
\operatorname{LBP}\left(g p_{x}, g p_{y}\right)=\sum_{p=1}^{p} S\left(g_{p}-g_{c}\right) * 2^{p}
$$

where gp and gc indicate the values of adjacent pixel and central pixel. LBP value of each pixel must be calculated based on the equation to generate the LBP Face image.

Figure 3: Illustration of LBP Coding

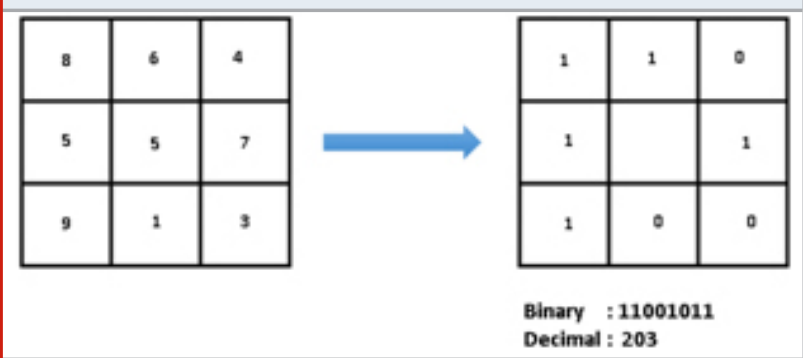

The figure 4, depicts the LBP texture descriptors of facial emotion happy, the output image shows more accurate values than that is obtained from grayscale.

Figure 4: Illustration of the input image converted into LBP

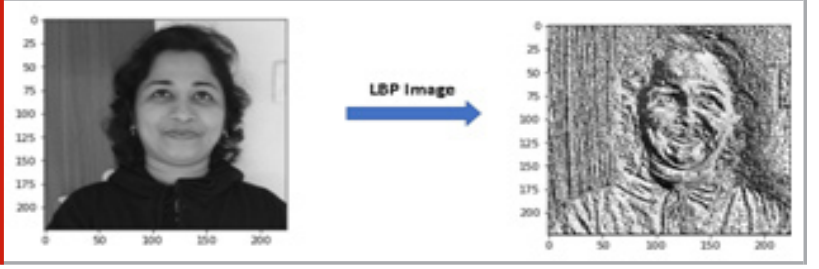

Datasets Used in Detail: Huge amount of labelled data is required to train a neural network. The popular datasets Cohn-Kanade (CK+) consists of 8363 images for testing and training.

The proposed system used the dataset FER 2013 to train models. The dataset consists of 35887 images, out of which 28709 labelled images belong to training set and the other 7178 images belong to test set. Table 2 gives the count of each emotion.

\begin{tabular}{|l|c|c|}
\hline \multirow{2}{*}{ Table 1. Dataset Description } \\
\hline \multirow{2}{*}{ Base } & \multicolumn{2}{|c|}{ Number of images } \\
\hline \multirow{2}{*}{ Training } & CK+ & 8000 \\
& FER 2013 & 28709 \\
& Home brewed & 100 \\
\hline Testing & CK+ & 150 \\
& FER 2013 & 7178 \\
& Home brewed & 23 \\
\hline
\end{tabular}

$\begin{aligned} & \text { Table 2. Count of Emotions in the FER } \\
& 2013 \text { Dataset }\end{aligned}$
\begin{tabular}{|l|c|c|} 
Sl. No & $\begin{array}{c}\text { Type of } \\
\text { Emotion }\end{array}$ & $\begin{array}{c}\text { No: of images } \\
\text { in dataset }\end{array}$ \\
\hline 1 & Happy & 7215 \\
\hline 2 & Surprise & 3171 \\
\hline 3 & Fear & 4097 \\
\hline 4 & Disgust & 436 \\
\hline 5 & Neutral & 4965 \\
\hline 6 & Sad & 4830 \\
\hline 7 & Angry & 3995 \\
\hline
\end{tabular}

Deep Convolutional Neural Network Model: The model is based on Sequential (DCNN) Model and has got 6 convolutional Layers with of batch normalizations applied in between, 3 layers of MaxPooling is added along with 4 dropouts and 2 dense layers. The Activation function used here is ReLU (Agarap, 2018). Batch Normalization is included to make the training faster. It also makes the complex networks simple and allows higher learning rate and easy initialization of weights. The ReLU (Rectified Linear Unit) activation function makes the evaluation quicker and increases the non -linearity in the images. The main advantage of using ReLU is that it does not activate all the neurons simultaneously. That is the neurons will get deactivated only if the result of linear transformation is less than zero. The ReLU function is computationally more efficient than Sigmoid function.

The dimensionality of the images is reduced with the help of MaxPooling, which will help to reduce the size drastically. It is done by unifying he pixel neighbourhood to a single value. Visualization of the out puts of convolution layer and extracted feature maps of a home-brewed image is shown in Figure 6a and Figure 
6b. The overfitting of training data is reduced by adding Dropout functions. The flatten layer is added to convert the image into 1Dimensional Array. That is the output from convolution layer was a $2 \mathrm{D}$ matrix. The features that are send to fully connected layer will generate the result.

Figure 5: Visualization of the output of first Convolution Layer

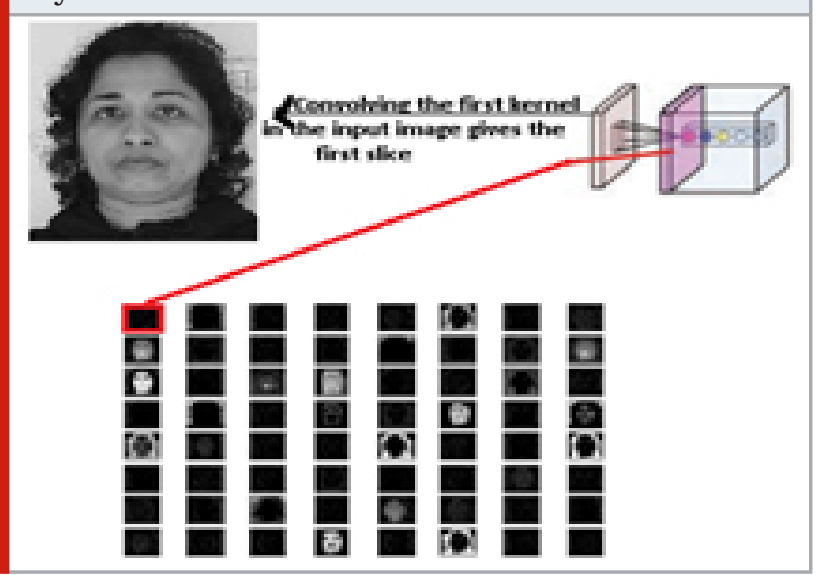

Figure 6: Visualization of the feature maps generated through various layers.

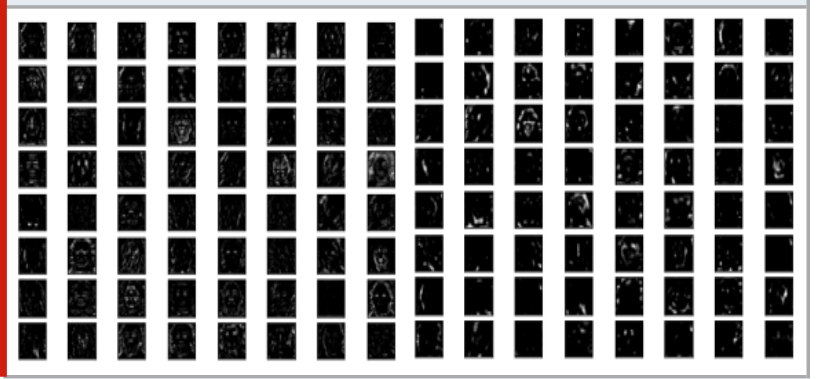

Figure 7: VGG 16 Convnet

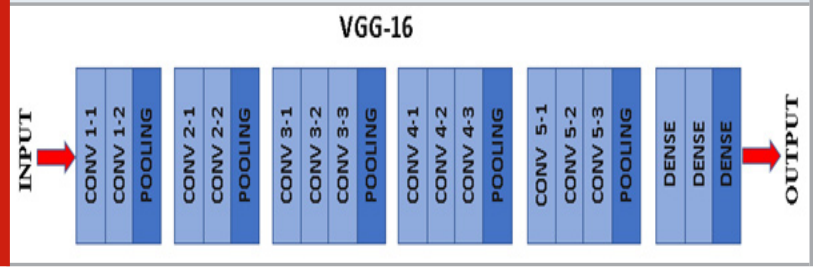

The Dense function is used to connect neurons in each layer. The output layer includes techniques like Dense and SoftMax (Chen, et al., 2018). SoftMax is a combination of multiple sigmoid, and here we use SoftMax for classification. The function also represents the probability for a data point of individual classes. The input images are trained in such a way to identify faces and classification of facial expression into various classes like Happy, Angry, Sad, Neutral, Disgust, Fear and Surprise. There are a total of seven steps included in the training process. This includes loading the dataset, pre-processing image, augmenting the feature vector (data), building and compiling the feature model, training and storing the model generated and finally validating the model. In the Real Time face detection, the image acquisition is done using a camera. From the captured image, the face is identified and bounded in a box and converted into binary pattern to specify as the feature vector and stored. The Local Binary Pattern Histogram (LBPH) helps in the detection of face using Viola -Jones Algorithm. The image pixels are stored with the threshold value for feature vector. These facial feature vectors are added with weights to form a neural network model.

Here the network architecture model used for facial expression and classification is VGG 16 CNN Model which is shown in Figure 7. Visual Geometry Group (VGG) was proposed by (Simonyan \& Zisserman, 2015). The image is passed through various convolutional layers arranged in a stack. All the hidden Layers are equipped with ReLU (Rectified Linear Unit Activation function). The spatial pooling is carried out by five Max-Pooling layers. Three Fully - Connected (FC) Layers, in that the first two have 4096 channels and the third consists of 1000 channels.

\section{RESULTS AND CONCLUSION}

FER 2013 dataset was found to be as good as other popular datasets in accuracy rate and performance. The best activation function seems to be ReLU [Rectified Linear Activation Function], which is the default activation function helps any model to learn faster and there is no issue of vanishing gradient problem like other activation functions. For Classification, Viola-Jones Haar Cascade classifier works well with OpenCV. So, a combination of the above three will help in developing a better FER System. Even though in the architecture the actual input to first convolution layer is 224X 224, in the proposed work it is taken with a resolution of 48 $\mathrm{X} 48$. The detected faces, which are rotation rectified in the input images are rescaled and reduced to $48 \mathrm{X} 48$ to make uniformity to all the images that are being trained and tested. The reduction in image area will help in accelerating the speed of FER. But sometimes it may result in losing facial information, that are acquired from LBP images. The comparison of face emotion recognition rate obtained in the proposed model with the State- of -art is shown in Table 3.

Table 3. Comparison of Emotion Recognition rate obtained in the proposed model with state-of-art literature.

\begin{tabular}{|l|c|c|}
\hline Data Set & Method & Accuracy \\
\hline CK+ & $\begin{array}{c}\text { (Shan, et al., 2009) } \\
\text { six classes }\end{array}$ & 89.1 \\
\hline & $\begin{array}{c}\text { (Jeni, et al., 2012) } \\
\text { six classes }\end{array}$ & 96.0 \\
\hline FER 2013 & Proposed method & 89.0 \\
\hline Home-brewed Data set & Proposed Method & 95.0 \\
\hline
\end{tabular}

The proposed work involves three different phases as Face Detection, Face Recognition and Emotion Classification 
Each phase is explained below for better understanding. Facial Expression recognition is built with CNN model where the facial features are extracted and classified based on VGG 16 model. The proposed work involves three different phases, in the first phase the image will be captured by a camera, and reads the image based on Open CV HAAR cascade detection and Viola Jones Algorithm. The RGB image that is captured is processed and converted into matrix whose values ranges from 0 to 255. In the second step, the trained model generated is tested to recognize the human face, here the image resolution is adjusted to perform computation easy as per CNN model. In the third phase classification of emotions are done. The plot of featured images from the dataset is as shown in figure 8 . The real time emotions classified in the proposed work are angry, sad, neutral, happy, disgust and surprise are shown in figures 9- 14. The emotion, fear cannot be predicted fine in the work.

Figure 8: Plot of features images from FER2013 dataset

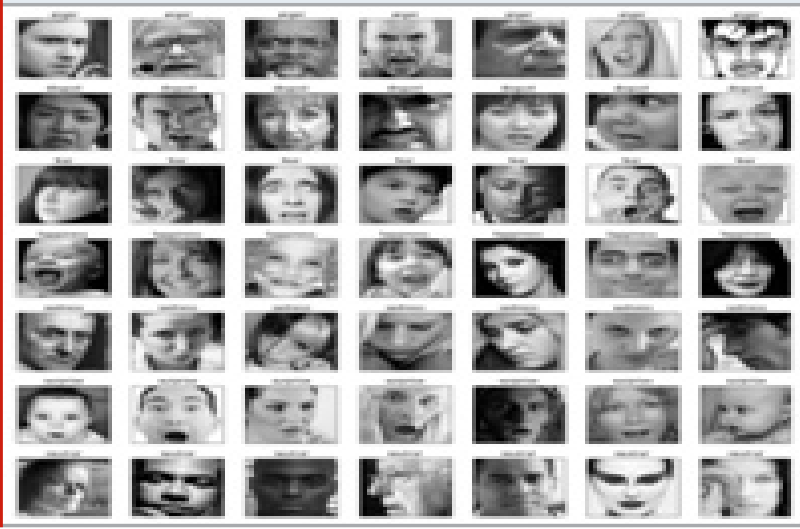

Figure 9: Face Emotion classified from the image is Anger

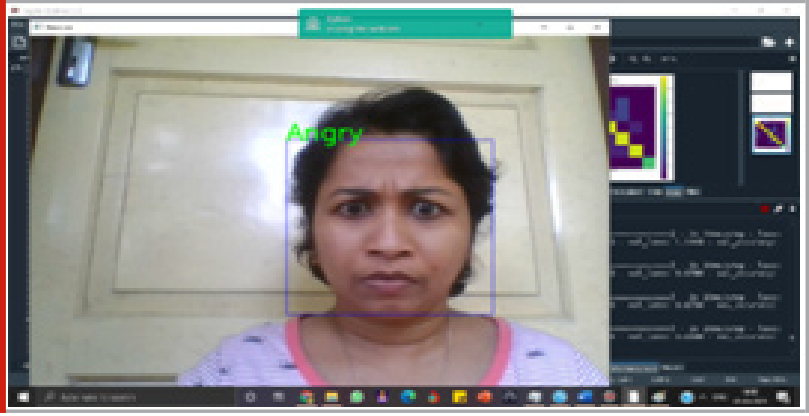

Figure 10: Emotion is detected as Sad

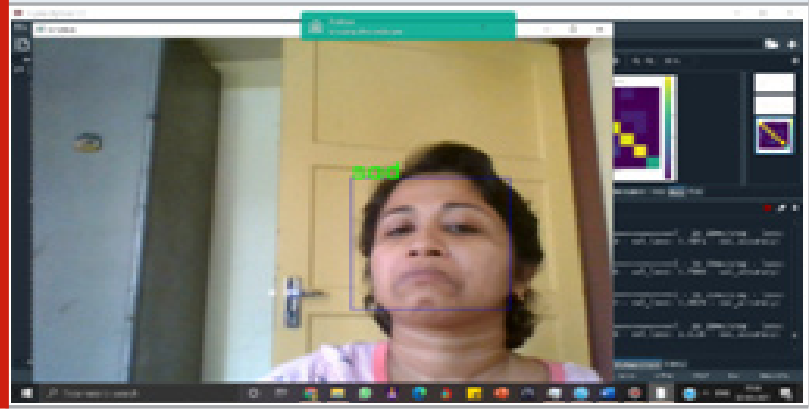

158
Figure 11: Emotion detected as disgust and in an untrained image, emotion is deteced as happy

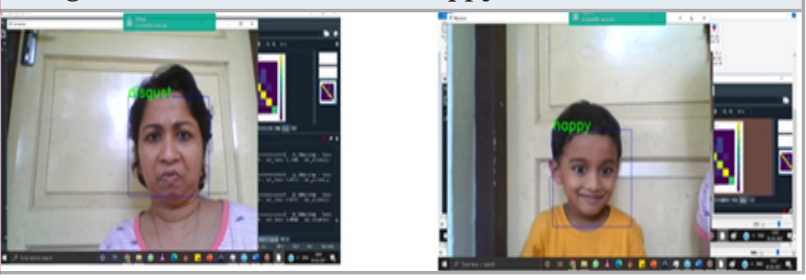

Figure 12: Two faces are identified in a single frame, with emotion detected as neutral.

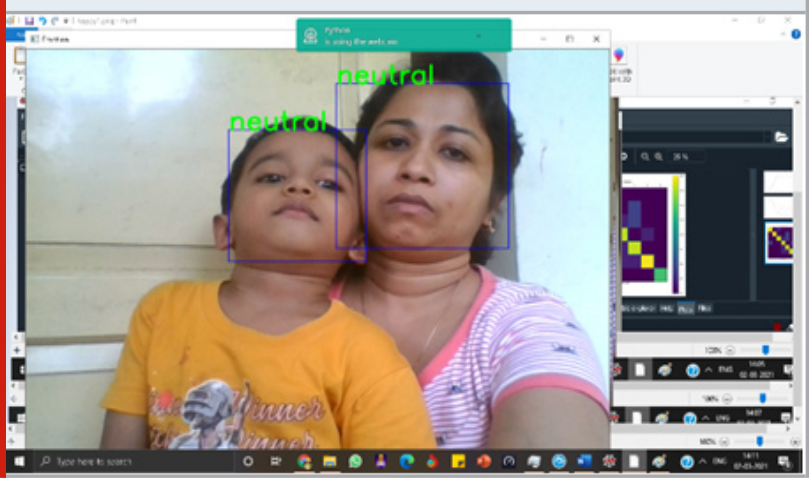

Figure 13: Emotion detected as Surprise

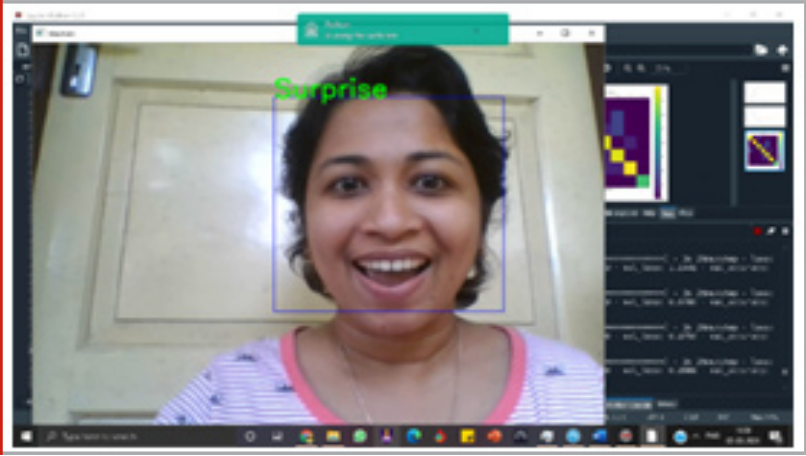

Figure 14: Untrained image-Emotion detected as Surprise.

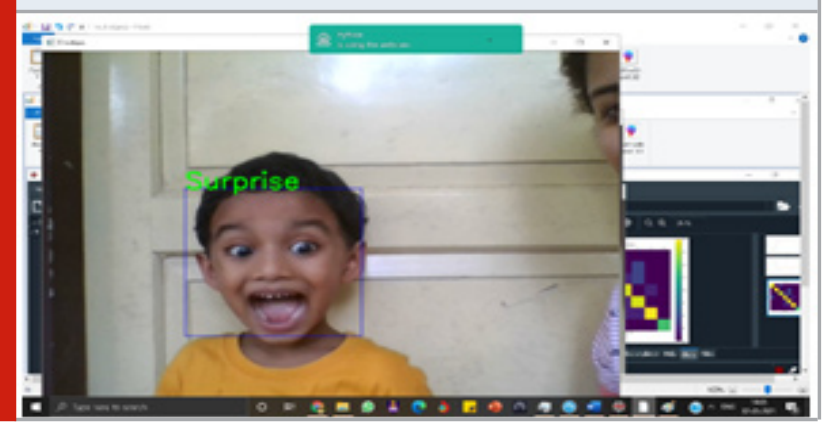

Figures 15 and 16 shows the confusion matrix obtained over the datasets FER 2013 and Home-brewed dataset. Figures 17 and 18 shows the accuracy and loss rates obtained in 50 epochs.

In the proposed work Convolutional neural network is implemented for Real time Facial Expression Recognition 
, and the model has been tested over a public dataset to assess the proposed model. A larger dataset FER2013, helps to improve model accuracy. The illumination and lighting conditions play an important role in image detection .In perfect laboratory condition (camera in the eye level, perfect lighting, subject with expression on face) the proposed work is compared with the Stateof-art literature and an accuracy of more than $90 \%$ is achieved, any deviaiton from the above condition results fall of accuracy. The CNN model used can vary in the number of layers included, but it is found that there is no deviation from achieved accuracy, as the number of epoch increase, it results in higher accuracy rate, but higher number of epochs may result in overfitting also.

Figure 15: Confusion Matrix during training on FER2013 dataset

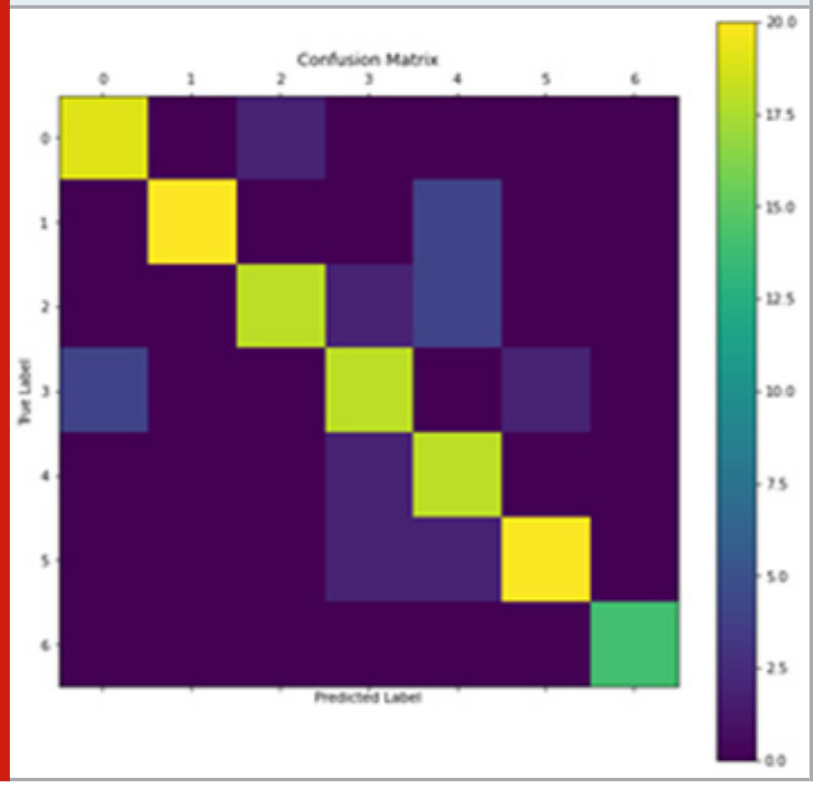

Figure 16: Confusion Matrix during training on Homebrewed dataset

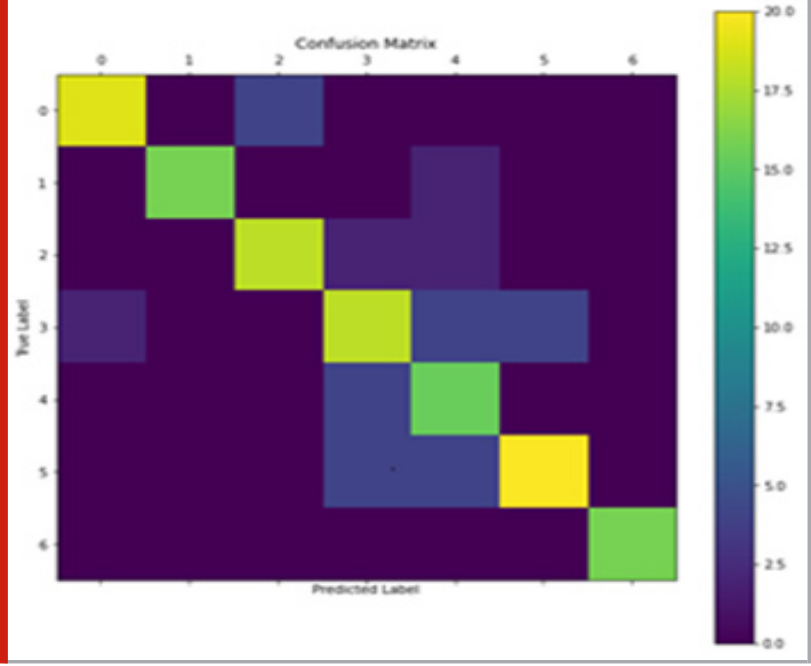

Facial Emotion Recognition is always a growing research area since it requires more and more accuracy in real-time image sensors. Effective Real-time monitoring will help in building very efficient applications that help to track lost objects, identify a person from a crowd in a dynamic environment. The emotion of a student undergoing digital learning can be predicted easily through this work. Open CV works better in developing real-time applications in IoT and found to be more productive and has high performance for face detection.

Figure 17: Plot of Accuracy rate in 50 epochs

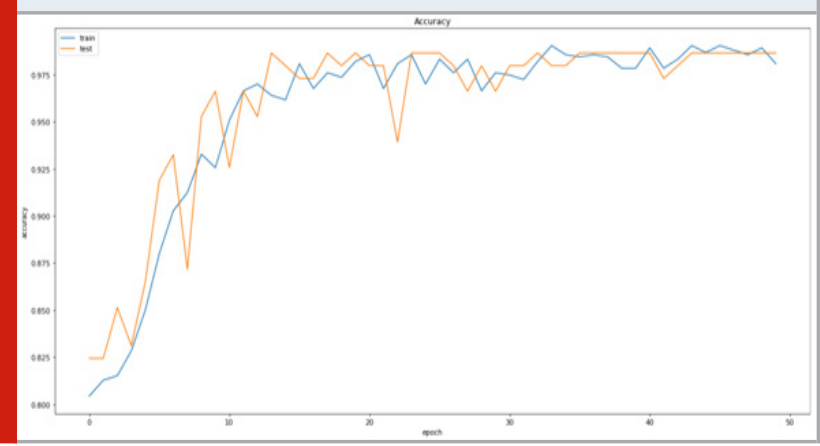

Figure 18: Plot of Loss rate in 50 epochs

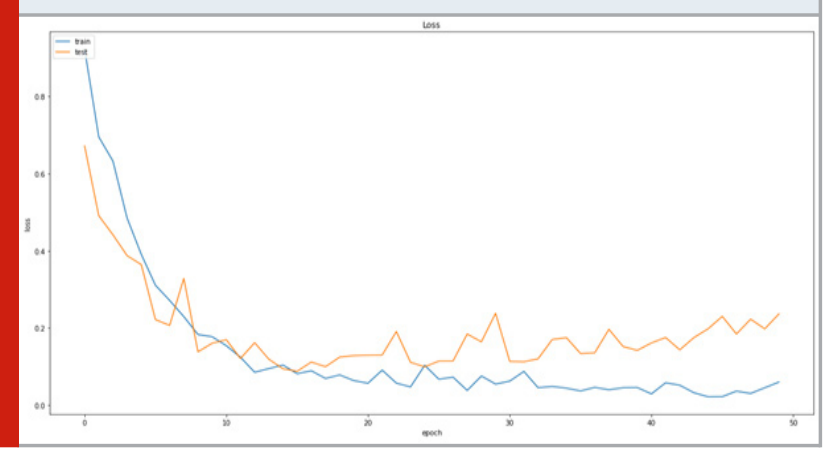

REFERENCES

Agarap, A. F., 2018. Deep Learning using Rectified Linear Units (ReLU). Neural and Evolutionary Computing.

Albert, M., 2008. Communication Without Words. In: 2. Edition, ed. Communication Theory. Routledge: Taylor Ct Francis, p. 8.

Anila, S. At Devarajan , N., 2012. Preprocessing technique for face recognition applications under varying illumination conditions. Global Journal of Computer Science and TechnologyGraphics \& Vision, 12(11), pp. 0975-4350.

Anitha , S. \& Radha, D. V., 2010. Comparision of image Preprocessing Techniques for Textile Texture Images. International Journal of Engineering Science and Technology, 2(12), p. 2(12).

Anon., n.d. In: s.l.:s.n.

Bartlett, M. S., Movellan, J. R. \&t Sejnowski, T. J., 2002. Face recognition by independent component analysis. Neural Networks, IEEE Transaction, pp. 1450-64. 
Belhumeur, P., Hespanha, J. \&t Kriegman, D., 1997. Eigenfaces vs. Fisherfaces: recognition using class specific linear projection. IEEE Transactions on Pattern Analysis and Machine Intelligence, 19(7), pp. 711 720.

Butalia, A., Ingle, M. \&t Kulkarni, P., 2012. Facial Expression Recognition for Security. International Journal of Modern Engineering Research, 2(4), pp. -1449-1453.

Chan, C.-H., Kittler, J. \&t Messer, K., 2007. Multi-scale Local Binary Pattern Histograms for Face Recognition. Advances in Biometrics, pp. 809-818.

Chen, L. et al., 2018. Softmax regression based deep sparse autoencoder network for facial emotion recognition in human-robot interaction. Information Sciences, Volume 428, pp. 49-61.

Chen, X., Yang, X., Wang, M. \&t Zou, J., 2017. Convolution neural network for automatic facial expression recognition. Sapporo, Japan, IEEE.

Darwin, C., 1872. The expression of emotions in animals and man. London: Murray.

Dureha, A., 2014. An Accurate Algorithm for Generating a Music Playlist. International Journal of Computer Applications (0975 - 8887), Volume 100(9).

Ekman, P., Friesen \& Wallace, V., 1971. Constants across cultures in the face and emotion.. Journal of Personality and Social Psychology, 17(2), pp. 124-129.

Eschbach, R., Marcu, G. \&t Rizzi, A., 2014. Color Imaging XIX: Displaying, Processing, Hardcopy, and Applications. s.l., spiedigitallibrary.

Fasel, B. \& Luettin, J., 2003. Automatic facial expression analysis: a survey. Pattern Recognition, 36(1), pp. 259275.

Haiyang, Z., 2011. Image Preprocessing Methods in Face Recognition. In: 2156-8464 Infona. s.l.:IEEE, pp. 978-1-4244-6554-5 , 978-1-4244-6553-8.

Jabid, T., Hasanul Kabir, M. \&t Chae, 0., 2010. Facial expression recognition using Local Directional Pattern (LDP). IEEE Xplore, Issue 2010 IEEE International Conference on Image Processing.

Jeni, L. A., Takacs, D. \& Lorincz, A., 2012. High quality facial expression recognition in video streams using shape related information only. Barcelona, Spain, 2011 IEEE International Conference on Computer Vision Workshops (ICCV Workshops).

Kumar, D. J., Shamsolmoali, P. \&t Sehdev, P., 2019. Extended deep neural network for facial emotion recognition. Pattern Recognition Letters, Volume 120, pp. 69-74.

Kumar, D. S. Et Gupta, A., 2015. Facial Expression Recognition: A Review. s.l., s.n.

Kumari, J., R, R. \&t Pooja, K., 2015. Facial Expression Recognition: A Survey. Procedia Computer Science,
Volume 58, pp. 486-491.

Kumbhar, P. P. Y. et al., 2017. Real Time Face Detection and Tracking Using. INTERNATIONAL JOURNAL FOR RESEARCH IN EMERGING SCIENCE AND TECHNOLOGY, 4(4).

Lucey, P. et al., 2010. The Extended Cohn-Kanade Dataset $(\mathrm{CK}+)$ : A complete dataset for action unit and emotion-specified expression. IEEE Xplore, Issue 2010 IEEE Computer Society Conference on Computer Vision and Pattern Recognition - Workshops, p. 11466835.

Mandal, M. K., Pandey, R. \&t Prasad, A. . B., 1998. Facial Expressions of Emotions and Schizophrenia: A Review. Schizophrenia Bulletin Journals, 24(3), p. 399-412.

Ojala, T., Pietikäinen, M. \&t Harwood, D., 1996. A comparative study of texture measures with classification based on featured distributions. Pattern Recognition, 29(1), pp. 51-59.

Ojala, T., Pietikainen, M. \&t Maenpaa, T., 2002. Multiresolution gray-scale and rotation invariant texture classification with local binary patterns. IEEE Explore, 24(7), pp. 971 - 987.

Openaire, C., 1998. The Japanese Female Facial Expression (JAFFE) Dataset. [Online] Available at: https://zenodo.org/record/3451524\#.YJ-XrKgzbIV [Accessed 2021].

Polikovsky, S., Kameda, Y. \& Ohta , Y., 2009. Facial Micro-Expressions Recognition using High Speed Camera. IET Digital Library, Volume 3rd International Conference on Imaging for Crime Detection and Prevention (ICDP 2009).

Shamsolmoali, P. et al., 2018. Deep convolution network for surveillance records super-resolution. SpringerLink Multimedia Tools and Applications, Volume 78, p. 23815-23829.

Shan, C., Gong, S. \&t McOwan, P. W., 2009. Facial expression recognition based on Local Binary Patterns: A comprehensive study. Image and Vision Computing, 27(6), pp. 803-816.

Shan, C., Gong, S. \&t McOwan, P. W., 2009. Facial expression recognition based on Local Binary Patterns: A comprehensive study. Image and Vision Computing, 27(6), pp. 803-816.

Shoayuan, X., Yang, C., Qian, L. \&t Jan, A., 2019. Emotion Recognition Using Convolutional Neural Networks. Electronic Imaging- Imaging and Multimedia Analytics in a Web and Mobile World, Society for Imaging Science and Technology(9), pp. 402-1-402-9. Simonyan, K. \& Zisserman, A., 2015. Very Deep Convolutional Networks for Large-Scale Image Recognition. Computer Vision and Pattern Recognition.

Sun, A. et al., 2018. Facial expression recognition using optimized active regions. Springer Open Human-centric 
Computing and Information Sciences, Volume 8.

Tong, Y., Chen, R. \&t Cheng, Y., 2014. Facial expression recognition algorithm using LGC based on horizontal and diagonal prior principle. Optik, 125(16), pp. 41864189.

Turabzadeh, S. et al., 2017. Real-time emotional state detection from facial expression on embedded devices. IEEE Xplore.

Turk, M. A. \&t Pentland, A. P., 1991. Face recognition using eigenfaces.. Computer Vision and Pattern Recognition. Proceedings IEEE Computer Society Conference, pp. 586-91.

University, T. R. I. C. M., 2021. Cohn-Kanade AU-
Coded Facial Expression Database. [Online] Available at: https://www.ri.cmu.edu/project/cohn-kanade-aucoded-facial-expression-database/ [Accessed 2021]. Wang, S. et al., 2010. A Natural Visible and Infrared Facial Expression Database for Expression Recognition and Emotion Inference. IEEE Xplore, 12(7), pp. 682 691.

Wang, Y.-Q., 2014. An Analysis of the Viola-Jones Face Detection Algorithm. IPOL Journal • Image Processing On Line, Volume 4, p. 128-148.

Zhang, Z. \& Zhang, J., 2006. A New Real-Time Eye Tracking for Driver Fatigue Detection. IEEE Xplore, Issue 2006 6th International Conference on ITS Telecommunications. 